\title{
On a system of fractional coupled hybrid Hadamard differential equations with terminal conditions
}

\author{
Karthikeyan Buvaneswari ${ }^{1}$, Panjaiyan Karthikeyan² and Dumitru Baleanu ${ }^{3,4,5^{*}}$ (B)
}

\section{"Correspondence:}

dumitru@cankaya.edu.tr

${ }^{3}$ Department of Mathematics, Cankaya University, Ankara, Turkey

${ }^{4}$ Institute of Space Sciences, 06530 Magurele-Bucharest, Romania Full list of author information is available at the end of the article

\section{Springer}

\begin{abstract}
In this manuscript, we study the existence of solutions for a coupled system of nonlinear hybrid differential equations of fractional order involving Hadamard derivative with nonlocal boundary conditions. By using suitable fixed point theorems we establish sufficient conditions for the existence result. An example is provided to illustrate our main result.
\end{abstract}

MSC: Primary 34A08; secondary 34B18

Keywords: Hadamard fractional derivative; Nonlocal conditions; Fixed point theorem; Existence

\section{Introduction}

Differential Equations of Fractional Order (DEFO) gain huge attention among scientists due to the applications which are not possible with integer order ordinary or partial differential equations. In recent days, researchers in various fields pay more attention on the existence of solutions of the aforesaid equations. In this regard, many literature surveys, some notable developments (refer[13, 14]), and significant studies are available in the theory of coupled systems with coupled boundary conditions in FDEs. For further references, one can refer to $[1,3,6-9,12,15,16,19]$. This is because the aforesaid equations include the perturbations of original differential equations in nonidentical ways.

The nonlinear hybrid Hadamard differential equations with initial conditions were discussed by Ahmad and Ntouyas in [4]. In 2015, Wang [22] proved the existence results for the impulsive systems with Hadamard derivative. For more details, the interested readers can refer to Wang et al. [21], where the authors used some classical methods to analyze the Ulam-Hyers stability and proved existence results for Hadamard fractional differential equations. Further, Samko et al. [20] proved the existence and uniqueness of the solution of Cauchy problems for FDE involving Hadamard derivatives in a nonsequential setting. In [17], the author studied the Hadamard-type fractional calculus.

We believe that only few literature studies are available which investigate coupled systems of FDEs of Hadamard type; see [23, 24] and the references therein. This paper will definitely fill up the gap between the theories and applications of Hadamard FDEs.

(c) The Author(s) 2020. This article is licensed under a Creative Commons Attribution 4.0 International License, which permits use sharing, adaptation, distribution and reproduction in any medium or format, as long as you give appropriate credit to the original author(s) and the source, provide a link to the Creative Commons licence, and indicate if changes were made. The images or other third party material in this article are included in the article's Creative Commons licence, unless indicated otherwise in a credit line to the material. If material is not included in the article's Creative Commons licence and your intended use is not permitted by statutory regulation or exceeds the permitted use, you will need to obtain permission directly from the copyright holder. To view a copy of this licence, visit http://creativecommons.org/licenses/by/4.0/. 
In[4], Ahmad et al. studied

$$
\left\{\begin{array}{l}
{ }_{H} D^{\alpha_{1}}\left(\frac{v(t)}{f_{1}(t, v(t))}\right)=g_{1}(t, v(t)), \quad 1 \leq t \leq T, 0<\alpha_{1} \leq 1, \\
H^{1-\left.\alpha_{1} v(t)\right|_{t=1}=\zeta}
\end{array}\right.
$$

In 2015, the authors of [5] investigated the nonlocal boundary value problems for hybrid FDEs and inclusions of Hadamard type as given below:

$$
\left\{\begin{array}{l}
D^{\alpha_{1}}\left(\frac{v(t)}{f_{1}(t, v(t))}\right)=g_{1}(t, v(t)), \quad 1 \leq t \leq e, 1<\alpha_{1} \leq 2, \\
v(1)=0, \quad v(e)=m(v),
\end{array}\right.
$$

Ahmad et al. [2] in 2017 focused on the issues related to initial and boundary value problems for fractional differential equations of Hadamard type and studied mixed initial value problems involving Hadamard derivatives.

The above mentioned works motivated us to study the following coupled Hadamard FDEs with the boundary conditions:

$$
\begin{cases}D^{\sigma_{1}}\left(\frac{\vartheta(t)}{f_{1}(t, \vartheta(t), v(t))}\right)=h_{1}(t, \vartheta(t), v(t)), & 1 \leq t \leq e, 1<\sigma_{1} \leq 2, \\ D^{\delta_{1}}\left(\frac{v(t)}{f_{2}(t, \vartheta(t), v(t))}\right)=h_{2}(t, \vartheta(t), v(t)), & 1 \leq t \leq e, 1<\delta_{1} \leq 2, \\ \vartheta(1)=0, \quad \vartheta(e)=m(\vartheta), & \\ v(1)=0, \quad v(e)=n(v), & \end{cases}
$$

where $D^{\sigma_{1}}, D^{\delta_{1}}$ are the Hadamard fractional derivatives of order $\sigma_{1}$ and $\delta_{1}$, respectively; $f_{i} \in \mathfrak{C}([1, e] \times \mathfrak{R} \times \mathfrak{R}, \mathfrak{R} \backslash\{0\})$ and $h_{i} \in \mathfrak{C}([1, e] \times \mathfrak{R} \times \mathfrak{R}, \mathfrak{R}), i=1,2, m, n: \mathfrak{C}([1, e], \mathfrak{R}) \rightarrow \mathfrak{R}$ are continuous functions.

The rest of the paper is organized as follows. In Sect. 2, some basic definitions and lemmas related to Hadamard fractional derivatives are presented. In Sect. 3, we establish sufficient conditions for the existence of the coupled solutions to (3), and in Sect. 4, we present a numerical example to illustrate our main result.

\section{Auxiliary results}

In this section we list some basic definitions and lemmas of fractional calculus.

We define the nonhomogeneous boundary value problems of coupled hybrid fractional differential equations of Hadamard type of the function space $\mathcal{X}=\mathfrak{C}([1, e], \Re)$ of continuous real valued functions $f_{1}:[1, e] \rightarrow \mathfrak{R}$. Clearly, $\mathcal{X}=\mathfrak{C}([1, e], \mathfrak{R})$ is a Banach space under the supremum norm

$$
\|\vartheta\|=\sup \{|\vartheta(t)|: t \in[1, e]\}
$$

which is again a Banach algebra with respect to the multiplication "." defined by

$$
(\vartheta \cdot v)(t)=\vartheta(t) \cdot v(t)
$$

The product space $\mathcal{E}=\mathcal{X} \times \mathcal{X}$ is a Banach space under the norm

$$
\|(\vartheta, v)\|=\|\vartheta\|+\|v\| .
$$


Then the normed linear space $(\mathcal{E},\|(\cdot, \cdot)\|)$ is a Banach space which further becomes a Banach algebra with respect to the multiplication "." defined by

$$
((\vartheta, v) \cdot(\bar{u}, \bar{v}))(t)=(\vartheta, v)(t) \cdot(\bar{u}, \bar{v})(t)=(\vartheta(t) \bar{u}(t), v(t) \bar{v}(t))
$$

for all $t \in[1, e]$, where $(\vartheta, v),(\bar{u}, \bar{v}) \in \mathcal{X} \times \mathcal{X}=\mathcal{E}$. The following result concerning this fact of algebraic structure of the product space $\mathcal{E}=\mathcal{X} \times \mathcal{X}$ is proved by Dhage in [11]. But first we need

Definition 2.1 ([18]) The Hadamard fractional derivative of order $\beta_{1}$ for a continuous function $h_{1}:[1, \infty] \rightarrow \mathfrak{R}$ is defined as

$$
D^{\beta_{1}} h_{1}(t)=\frac{1}{\Gamma\left(n-\beta_{1}\right)}\left(t \frac{d}{d t}\right)^{n} \int_{1}^{t}\left(\ln \frac{t}{s}\right)^{n-\beta_{1}-1} \frac{h_{1}(s)}{s} d s, \quad n-1<\beta_{1}<n,
$$

where $n=\left[\beta_{1}\right]+1,\left[\beta_{1}\right]$ denotes the integer part of the real number $\beta_{1}$ and $\ln (\cdot)=\log _{e}(\cdot)$.

Definition $2.2([18])$ The Hadamard fractional integral of order $\beta_{1}$ for a function $h_{1}$ : $[1, \infty) \rightarrow \mathfrak{R}$ is defined as

$$
I^{\beta_{1}} h_{1}(t)=\frac{1}{\Gamma\left(\beta_{1}\right)} \int_{1}^{t}\left(\ln \frac{t}{s}\right)^{\beta_{1}-1} \frac{h_{1}(s)}{s} d s, \quad \beta_{1}>0, t>1,
$$

provided the integral exists.

Definition 2.3 (Dhage [11]) Let $\mathcal{E}$ be a Banach space. An operator $\mathcal{T}: \mathcal{E} \rightarrow \mathcal{E}$ is called Lipschitz if there exists a constant $L_{\mathcal{T}}>0$ such that

$$
\|\mathcal{T}(\vartheta)-\mathcal{T}(v)\| \leq L_{\mathcal{T}}\|\vartheta-v\|
$$

for all elements $\vartheta, v \in \mathcal{X}$.

Lemma 2.4 (Dhage [11]) The product space $\mathcal{E}$ is a Banach algebra with respect to the norm $\|(\cdot, \cdot)\|$ and the multiplication "." defined by (6) and (7), respectively.

Proof Let $(\vartheta, v)$ and $(\bar{u}, \bar{v})$ be any two elements of $\mathcal{E}$ then, by definition of the norm $\|(\cdot, \cdot)\|$ and the multiplication "." in $\mathcal{E}$, we obtain

$$
\|(\vartheta, v) \cdot(\bar{u}, \bar{v})\|=\|(\vartheta \bar{u}, v \bar{v})\|=\|\vartheta \bar{u}\|+\|v \bar{v}\| \leq\|\vartheta\|\|\bar{u}\|+\|v\|\|\bar{v}\|
$$

and

$$
\|(\vartheta, v)\|\|(\bar{u}, \bar{v})\|=[\|\vartheta\|+\|\bar{u}\|][\|v\|+\|\bar{v}\|] .
$$

Now from (8) and (9), it follows that

$$
\|((\vartheta, v)(\bar{u}, \bar{v}))\| \leq\|(\vartheta, v)\|\|(\bar{u}, \bar{v})\| .
$$


This shows that $(\mathcal{E},\|(\cdot, \cdot)\|)$ is a Banach algebra and the proof of lemma is complete.

We employ the following fixed point theorem as a basic tool for proving our main existence result for a coupled system of the proposed problem (3).

Theorem 2.5 (Dhage [10]) Let $\mathcal{S}$ be a nonempty subset of a Banach algebra, which is bounded, convex and closed. Assume also that the operators $\mathcal{A}$ and $\mathcal{B}$ are such that $\mathcal{A}: \mathcal{X} \rightarrow \mathcal{X}$ and $\mathcal{B}: \mathcal{S} \rightarrow \mathcal{X}$, and satisfy the following conditions:

$\left(a_{1}\right) \mathcal{A}$ is Lipschitzian with $L_{\mathcal{A}}$ as a Lipschitz constant,

$\left(a_{2}\right) \mathcal{B}$ is completely continuous,

$\left(a_{3}\right) \vartheta=\mathcal{A} \vartheta \mathcal{B} v \Longrightarrow \forall v \in S \Longrightarrow \vartheta \in \mathcal{S}$, and

$\left(a_{4}\right) M_{\mathcal{B}}<1$, where $M_{\mathcal{B}}=\|\mathcal{B}(S)\|=\sup \{\|\mathcal{B} \vartheta\|: \vartheta \in \mathcal{S}\}$.

Then the operator equation $\vartheta=\mathcal{A} \vartheta \mathcal{B} \vartheta$ has a solution in $S$.

\section{Existence result}

To prove the main existence result for a mild coupled solution of problem (3), we need to prove the following lemma.

Lemma 3.1 Given $\psi_{1}, \psi_{2} \in \mathfrak{C}([1, e] \times \mathfrak{R} \times \mathfrak{R} \rightarrow \mathfrak{R})$, the integral solution of the boundary value problem

$$
\left\{\begin{array}{l}
D^{\omega_{1}}\left(\frac{\vartheta(t)}{\psi_{1}(t, \vartheta(t), v(t))}\right)=\psi_{2}(t), \quad 1 \leq t \leq e, 1<\omega_{1} \leq 2, \\
\vartheta(1)=0, \quad \vartheta(e)=m(\vartheta)
\end{array}\right.
$$

is given by

$$
\begin{aligned}
\vartheta(t)= & \psi_{1}(t, \vartheta(t), v(t)) \times\left[\frac{1}{\Gamma\left(\omega_{1}\right)} \int_{1}^{t}\left(\ln \frac{t}{s}\right)^{\omega_{1}-1} \frac{\psi_{2}(s)}{s} d s\right. \\
& \left.+(\ln t)^{\omega_{1}-1}\left(\frac{m(\vartheta)}{\psi_{1}(e, m(\vartheta), v(e))}-\frac{1}{\Gamma\left(\omega_{1}\right)} \int_{1}^{e}\left(\ln \frac{e}{s}\right)^{\omega_{1}-1} \frac{\psi_{2}(s)}{s} d s\right)\right] .
\end{aligned}
$$

Proof The solution of equation (10) can be written as

$$
\begin{aligned}
\vartheta(t)= & \psi_{1}(t, \vartheta(t), v(t))\left[\frac{1}{\Gamma\left(\omega_{1}\right)} \int_{1}^{t}\left(\ln \frac{t}{s}\right)^{\omega_{1}-1} \frac{\psi_{2}(s)}{s} d s+c_{1}(\ln t)^{\omega_{1}-1}\right. \\
& \left.+c_{2}(\ln t)^{\omega_{1}-2}\right]
\end{aligned}
$$

where the arbitrary constants $c_{1}, c_{2} \in \mathfrak{R}$ and the boundary conditions given in (10) give $c_{2}=0$ and

$$
c_{1}=(\ln t)^{\omega_{1}-1}\left(\frac{m(\vartheta)}{\psi_{1}(e, m(\vartheta), v(e))}-\frac{1}{\Gamma\left(\omega_{1}\right)} \int_{1}^{e}\left(\ln \frac{e}{s}\right)^{\omega_{1}-1} \frac{\psi_{2}(s)}{s} d s\right) .
$$


Substituting these values of $c_{1}$ and $c_{2}$ into (12), we get

$$
\begin{aligned}
\vartheta(t)= & \psi_{1}(t, \vartheta(t), v(t)) \times\left[\frac{1}{\Gamma\left(\omega_{1}\right)} \int_{1}^{t}\left(\ln \frac{t}{s}\right)^{\omega_{1}-1} \frac{\psi_{2}(s)}{s} d s\right. \\
& \left.+(\ln t)^{\omega_{1}-1}\left(\frac{m(\vartheta)}{\psi_{1}(e, m(\vartheta), v(e))}-\frac{1}{\Gamma\left(\omega_{1}\right)} \int_{1}^{e}\left(\ln \frac{e}{s}\right)^{\omega_{1}-1} \frac{\psi_{2}(s)}{s} d s\right)\right] .
\end{aligned}
$$

Now, the proof is finished.

Definition 3.2 A solution $\vartheta \in \mathfrak{C}([1, e], \mathfrak{R}, \mathfrak{R})$ of the hybrid Hadamard fractional differential equation in (11) is called a mild solution of the FDE (10) defined on $[1, e]$. Similarly, a mild coupled solution $(\vartheta, v) \in \mathfrak{C}([1, e], \mathfrak{R}) \times \mathfrak{C}([1, e], \mathfrak{R})$ of a system of the coupled Hadamard differential equation (3) is defined on $[1, e]$.

Theorem 3.3 Consider the following hypotheses $(\mathrm{H} 1)$ to $(\mathrm{H} 4)$ :

(H1) For the continuous functions $f_{i}:[1, e] \times \mathfrak{R} \times \mathfrak{R} \rightarrow \mathfrak{R} \backslash\{0\}, i=1,2$ with constants $L_{f_{1}}>0$ and $L_{f_{2}}>0$, we have

$$
\mid f_{1}\left(t, \vartheta, v-f_{1}(t, \bar{\vartheta}, \bar{v}) \mid \leq L_{f_{1}}(|\vartheta-\bar{\vartheta}|+|v-\bar{v}|),\right.
$$

and

$$
\left|f_{2}(t, \vartheta, v)-f_{2}(t, \bar{\vartheta}, \bar{v})\right| \leq L_{f_{2}}(|\vartheta-\bar{\vartheta}|+|v-\bar{v}|)
$$

for all $t \in[1, e]$ and $\vartheta, \bar{\vartheta}, v, \bar{v} \in \mathfrak{R}$.

(H2) There exist constants $M_{1}>0$ and $M_{2}>0$ such that

$$
\left|\frac{m(\vartheta)}{f_{1}(e, m(\vartheta), n(v))}\right| \leq M_{1} \text { and }\left|\frac{n(v)}{f_{2}(e, m(\vartheta), n(v))}\right| \leq M_{2}
$$

and

$$
F_{0}=\sup _{t \in[1, e]}\left|f_{1}(t, 0,0)\right|, \quad G_{0}=\sup _{t \in[1, e]}\left|f_{2}(t, 0,0)\right| .
$$

(H3) There exist constants $M_{h_{i}}>0$ such that

$$
\left|h_{i}(t, \vartheta, v)\right| \leq M_{h_{i}}
$$

for all $(t, \vartheta, v) \in[1, e] \times \mathfrak{R} \times \mathfrak{R}$ and $i=1,2$.

(H4) The constants in hypotheses (H1) through (H3) satisfy the following conditions:

$$
\Omega=L_{f_{1}}\left(M_{1}+\frac{2 M_{h_{1}}}{\Gamma\left(\sigma_{1}+1\right)}\right)+L_{f_{2}}\left(M_{2}+\frac{2 M_{h_{2}}}{\Gamma\left(\delta_{1}+1\right)}\right)<1
$$

If these conditions hold, then the coupled system (3) has a coupled solution defined on $[1, e] \times[1, e]$. 
Proof Using Lemma 3.1 for (3), we get

$$
\begin{aligned}
\vartheta(t)= & f_{1}(t, \vartheta(t), v(t)) \times\left[\frac{1}{\Gamma\left(\sigma_{1}\right)} \int_{1}^{t}\left(\ln \frac{t}{s}\right)^{\sigma_{1}-1} \frac{h_{1}(s)}{s} d s\right. \\
& \left.+(\ln t)^{\sigma_{1}-1}\left(\frac{m(\vartheta)}{f_{1}(e, m(\vartheta), n(v))}-\frac{1}{\Gamma\left(\sigma_{1}\right)} \int_{1}^{e}\left(\ln \frac{e}{s}\right)^{\sigma_{1}-1} \frac{h_{1}(s)}{s} d s\right)\right]
\end{aligned}
$$

and

$$
\begin{aligned}
z(t)= & f_{2}(t, \vartheta(t), v(t)) \times\left[\frac{1}{\Gamma\left(\delta_{1}\right)} \int_{1}^{t}\left(\ln \frac{t}{s}\right)^{\delta_{1}-1} \frac{h_{2}(s)}{s} d s\right. \\
& \left.+(\ln t)^{\delta_{1}-1}\left(\frac{n(v)}{f_{2}(e, m(\vartheta), n(v))}-\frac{1}{\Gamma\left(\delta_{1}\right)} \int_{1}^{e}\left(\ln \frac{e}{s}\right)^{\delta_{1}-1} \frac{h_{2}(s)}{s} d s\right)\right] .
\end{aligned}
$$

Choose

$$
\rho \geq \frac{F_{0}\left(M_{1}+\frac{2 M_{h_{1}}}{\Gamma\left(\sigma_{1}+1\right)}\right)+G_{0}\left(M_{2}+\frac{2 M_{h_{2}}}{\Gamma\left(\delta_{1}+1\right)}\right)}{1-\Omega}
$$

and define a subset $\mathcal{S}$ of the Banach space $\mathcal{X} \times \mathcal{X}$ by

$$
\mathcal{S}=\{(\vartheta, v) \in \mathcal{X} \times \mathcal{X}:\|(\vartheta, v)\| \leq \rho\}
$$

Clearly, $\mathcal{S}$ is a closed, convex and bounded subset of the Banach space $\mathcal{E}=\mathcal{X} \times \mathcal{X}$. Define the operators $\mathcal{A}=\left(\mathcal{A}_{1}, \mathcal{A}_{2}\right): \mathcal{E} \rightarrow \mathcal{E}$ and $\mathcal{B}=\left(\mathcal{B}_{1}, \mathcal{B}_{2}\right): \mathcal{S} \rightarrow \mathcal{E}$ by

$$
\begin{aligned}
& \left\{\begin{aligned}
\mathcal{A}_{1}(\vartheta, v)= & f_{1}(t, \vartheta(t), v(t)), \quad t \in[1, e], \\
\mathcal{A}_{2}(\vartheta, v)= & f_{2}(t, \vartheta(t), v(t)), \quad t \in[1, e]
\end{aligned}\right. \\
& \left\{\begin{aligned}
\mathcal{B}_{1}(\vartheta, v)= & {\left[\frac{1}{\Gamma\left(\sigma_{1}\right)} \int_{1}^{t}\left(\ln \frac{t}{s}\right)^{\sigma_{1}-1} \frac{h_{1}(s)}{s} d s\right.} \\
& \left.+(\ln t)^{\sigma_{1}-1}\left(\frac{m(\vartheta)}{f_{1}(e, m(\vartheta), n(v))}-\frac{1}{\Gamma\left(\sigma_{1}\right)} \int_{1}^{e}\left(\ln \frac{e}{s}\right)^{\sigma_{1}-1} \frac{h_{1}(s)}{s} d s\right)\right] \\
\mathcal{B}_{2}(\vartheta, v)= & {\left[\frac{1}{\Gamma\left(\delta_{1}\right)} \int_{1}^{t}\left(\ln \frac{t}{s}\right)^{\delta_{1}-1} \frac{h_{2}(s)}{s} d s\right.} \\
& \left.+(\ln t)^{\delta_{1}-1}\left(\frac{n(v)}{f_{2}(e, m(\vartheta), n(v))}-\frac{1}{\Gamma\left(\delta_{1}\right)} \int_{1}^{e}\left(\ln \frac{e}{s}\right)^{\delta_{1}-1} \frac{h_{2}(s)}{s} d s\right)\right] .
\end{aligned}\right.
\end{aligned}
$$

Now, the system of equations in (13) and (14) becomes

$$
\mathcal{A}(\vartheta, v)(t) \mathcal{B}(\vartheta, v)(t)=(\vartheta, v)(t), \quad t \in[1, e],
$$

which further, in view of the multiplication (7) of two elements in $\mathcal{E}$, yields

$$
\left(\mathcal{A}_{1}(\vartheta, v)(t) \mathcal{B}_{1}(\vartheta, v)(t), \mathcal{A}_{2}(\vartheta, v)(t) \mathcal{B}_{2}(\vartheta, v)(t)\right)=(\vartheta, v)(t), \quad t \in[1, e] .
$$

This further implies that

$$
\begin{cases}\mathcal{A}_{1}(\vartheta, v)(t) \mathcal{B}_{1}(\vartheta, v)(t)=\vartheta(t), & t \in[1, e], \\ \mathcal{A}_{2}(\vartheta, v)(t) \mathcal{B}_{2}(\vartheta, v)(t)=v(t), & t \in[1, e] .\end{cases}
$$


Now we prove that the operators $\mathcal{A}$ and $\mathcal{B}$ satisfy the conditions of Theorem 2.5 in a series of steps.

Step I. First we show that $\mathcal{A}=\left(\mathcal{A}_{1}, \mathcal{A}_{2}\right)$ is Lipschitz on $\mathcal{E}$ with constant $\left(L_{f_{1}}+L_{f_{2}}\right)$. Let $(\vartheta, \nu),(\bar{\vartheta}, \bar{v}) \in \mathcal{E}$ be arbitrary. Then, using $(\mathrm{H} 1)$, we have

$$
\begin{aligned}
\mid \mathcal{A}_{1}(\vartheta, v)(t)-\mathcal{A}_{1}(\bar{\vartheta}, \bar{v})(t) & =\left|f_{1}(t, \vartheta(t), v(t))-f_{1}(t, \bar{\vartheta}(t), \bar{v}(t))\right| \\
& \leq L_{f_{1}}(|\vartheta(t)-\bar{\vartheta}(t)|+|v(t)-\bar{v}(t)|) \\
& \leq L_{f_{1}}(\|\vartheta-\bar{\vartheta}\|+\|v-\bar{v}\|)
\end{aligned}
$$

for all $t \in[1, e]$. Taking the supremum over $t$, we obtain

$$
\left\|\mathcal{A}_{1}(\vartheta, v)-\mathcal{A}_{1}(\bar{\vartheta}, \bar{v})\right\| \leq L_{f_{1}}(\|\vartheta-\bar{\vartheta}\|+\|v-\bar{v}\|)
$$

for all $(\vartheta, v),(\bar{\vartheta}, \bar{v}) \in \mathcal{E}$. Similarly, we obtain

$$
\left\|\mathcal{A}_{2}(\vartheta, v)-\mathcal{A}_{2}(\bar{\vartheta}, \bar{v})\right\| \leq L_{f_{2}}(\|\vartheta-\bar{\vartheta}\|+\|v-\bar{v}\|)
$$

for all $(\vartheta, v),(\bar{\vartheta}, \bar{v}) \in \mathcal{E}$. Therefore, by definition of the operator $\mathcal{A}$, we obtain

$$
\begin{aligned}
\| & \mathcal{A}(\vartheta, v)-\mathcal{A}(\bar{\vartheta}, \bar{v}) \| \\
& =\left\|\left(\mathcal{A}_{1}(\vartheta, v), \mathcal{A}_{2}(\vartheta, v)\right)-\left(\mathcal{A}_{1}(\bar{\vartheta}, \bar{v}), \mathcal{A}_{2}(\bar{\vartheta}, \bar{v})\right)\right\| \\
& =\left\|\left(\mathcal{A}_{1}(\vartheta, v)-\mathcal{A}_{1}(\bar{\vartheta}, \bar{v}), \mathcal{A}_{2}(\vartheta, v)-\mathcal{A}_{2}(\bar{\vartheta}, \bar{v})\right)\right\| \\
& \leq\left\|\mathcal{A}_{1}(\vartheta, v)-\mathcal{A}_{1}(\bar{\vartheta}, \bar{v})\right\|+\left\|\mathcal{A}_{2}(\vartheta, v)-\mathcal{A}_{2}(\bar{\vartheta}, \bar{v})\right\| \\
& \leq L_{f_{1}}(\|\vartheta-\bar{\vartheta}\|+\|v-\bar{v}\|)+L_{f_{2}}(\|\vartheta-\bar{\vartheta}\|+\|v-\bar{v}\|) \\
& =\left(L_{f_{1}}+L_{f_{2}}\right)(\|\vartheta-\bar{\vartheta}\|+\|v-\bar{v}\|),
\end{aligned}
$$

for all $(\vartheta, v),(\bar{\vartheta}, \bar{v}) \in \mathcal{E}$, where $L_{\mathcal{A}}=\left(L_{f_{1}}+L_{f_{2}}\right)$.

Step II. In this step we prove that $\mathcal{B}=\left(\mathcal{B}_{1}, \mathcal{B}_{2}\right)$ from $\mathcal{S}$ into $\mathcal{E}$ is a compact and continuous operator. For continuity of $\mathcal{B}$, take any sequence of points $\left(\vartheta_{n}, v_{n}\right)$ in $\mathcal{S}$ converging to a point $(\vartheta, v) \in \mathcal{S}$. Then, by Lebesgue dominated convergence theorem, we have

$$
\begin{aligned}
\lim _{n \rightarrow \infty} & \mathcal{B}_{1}\left(\vartheta_{n}, v_{n}\right)(t) \\
= & \lim _{n \rightarrow \infty}\left[\frac{1}{\Gamma\left(\sigma_{1}\right)} \int_{1}^{t}\left(\ln \frac{t}{s}\right)^{\sigma_{1}-1} \frac{h_{1}\left(s, \vartheta_{n}(s), v_{n}(s)\right)}{s} d s\right. \\
& +(\ln t)^{\sigma_{1}-1}\left(\frac{m(\vartheta)}{f_{1}(e, m(x), n(v))}\right. \\
& \left.\left.-\frac{1}{\Gamma\left(\sigma_{1}\right)} \int_{1}^{e}\left(\ln \frac{e}{s}\right)^{\sigma_{1}-1} \frac{h_{1}\left(s, \vartheta_{n}(s), v_{n}(s)\right)}{s} d s\right)\right] \\
= & \frac{1}{\Gamma\left(\sigma_{1}\right)} \int_{1}^{t}\left(\ln \frac{e}{s}\right)^{\sigma_{1}-1} \lim _{n \rightarrow \infty} \frac{h_{1}\left(s, \vartheta_{n}(s), v_{n}(s)\right)}{s} d s \\
& +(\ln t)^{\sigma_{1}-1}\left(\frac{m(\vartheta)}{f_{1}(e, m(x), n(v))}\right.
\end{aligned}
$$




$$
\begin{aligned}
& \left.-\frac{1}{\Gamma\left(\sigma_{1}\right)} \int_{1}^{e}\left(\ln \frac{e}{s}\right)^{\sigma_{1}-1} \lim _{n \rightarrow \infty} \frac{h_{1}\left(s, \vartheta_{n}(s), v_{n}(s)\right)}{s} d s\right) \\
= & \frac{1}{\Gamma\left(\sigma_{1}\right)} \int_{1}^{t}\left(\ln \frac{t}{s}\right)^{\sigma_{1}-1} \frac{h_{1}\left(s, \vartheta_{n}(s), v(s)\right)}{s} d s \\
& +(\ln t)^{\sigma_{1}-1}\left(\frac{m(\vartheta)}{f_{1}(e, m(\vartheta), n(v))}\right. \\
& \left.-\frac{1}{\Gamma\left(\sigma_{1}\right)} \int_{1}^{e}\left(\ln \frac{e}{s}\right)^{\sigma_{1}-1} \frac{h_{1}(s, \vartheta(s), v(s))}{s} d s\right) \\
= & \mathcal{B}_{1}(\vartheta, v)(t)
\end{aligned}
$$

for all $t \in[1, e]$. Similarly, we prove

$$
\lim _{n \rightarrow \infty} \mathcal{B}_{2}\left(\vartheta_{n}, v_{n}\right)(t)=\mathcal{B}_{2}(\vartheta, v)(t)
$$

for all $t \in[1, e]$. Hence $\mathcal{B}\left(\vartheta_{n}, v_{n}\right)=\left(\mathcal{B}_{1}\left(\vartheta_{n}, v_{n}\right) ; \mathcal{B}_{2}\left(\vartheta_{n}, v_{n}\right)\right)$ converges to $\mathcal{B}(\vartheta, v)$ pointwise on $[1, e]$. This shows that $\mathcal{B}$ is continuous.

Next, we prove $\mathcal{B}$ is a compact operator on $S$. Let $(\vartheta, v) \in S$ be any point. Then, using (H4), we have

$$
\begin{aligned}
\left|\mathcal{B}_{1}(\vartheta, v)\right|= & \mid \frac{1}{\Gamma\left(\sigma_{1}\right)} \int_{1}^{t}\left(\ln \frac{t}{s}\right)^{\sigma_{1}-1} \frac{h_{1}(s, \vartheta(s), v(s))}{s} d s \\
& +(\ln t)^{\sigma_{1}-1}\left(\frac{m(\vartheta)}{f_{1}(e, m(\vartheta), n(v))}\right. \\
& \left.-\frac{1}{\Gamma\left(\sigma_{1}\right)} \int_{1}^{e}\left(\ln \frac{e}{s}\right)^{\sigma_{1}-1} \frac{h_{1}(s, \vartheta(s), v(s))}{s} d s\right) \mid \\
\leq & \frac{M_{h_{1}}}{\Gamma\left(\sigma_{1}\right)} \int_{1}^{t}\left(\ln \frac{t}{s}\right)^{\sigma_{1}-1} \frac{1}{s} d s+M_{1}+\frac{M_{h_{1}}}{\Gamma\left(\sigma_{1}\right)} \int_{1}^{e}\left(\ln \frac{e}{s}\right)^{\sigma_{1}-1} \frac{1}{s} d s \\
\leq & \frac{2}{\Gamma\left(\sigma_{1}+1\right)} M_{h_{1}}+M_{1}
\end{aligned}
$$

for all $t \in[1, e]$.

Taking the supremum for the above inequality, we get

$$
\left\|\mathcal{B}_{1}(\vartheta, v)\right\| \leq \frac{2}{\Gamma\left(\sigma_{1}+1\right)} M_{h_{1}}+M_{1}
$$

for all $(\vartheta, v) \in \mathcal{E}$. Hence $\mathcal{B}_{1}$ is a uniformly bounded operator on $\mathcal{S}$. In a similar way, we can prove $\mathcal{B}_{2}$ is also uniformly bounded operator on $\mathcal{S}$. Hence $\mathcal{B}$ is a uniformly bounded operator on $\mathcal{S}$.

Next to prove that $\left\{\mathcal{B}\left(\vartheta_{n}, v_{n}\right)\right\}$ is an equicontinuous sequence of functions in $\mathcal{E}$, assume $\tau_{1}, \tau_{2} \in[1, e]$, provided $\tau_{1}<\tau_{2}$, then

$$
\begin{aligned}
& \left|\mathcal{B}_{1}(\vartheta, v)\left(\tau_{1}\right)-\mathcal{B}_{1}(\vartheta, v)\left(\tau_{2}\right)\right| \\
& \quad \leq \frac{M_{h_{1}}}{\Gamma\left(\sigma_{1}\right)}\left|\int_{1}^{\tau_{1}}\left(\ln \frac{\tau_{1}}{s}\right)^{\sigma_{1}-1} \frac{1}{s} d s-\int_{1}^{\tau_{2}}\left(\ln \frac{\tau_{2}}{s}\right)^{\sigma_{1}-1} \frac{1}{s} d s\right|
\end{aligned}
$$




$$
\begin{aligned}
& \quad+\left(\frac{M_{h_{1}}+\left|\left(\ln \tau_{2}\right)^{\sigma_{1}-1}-\left(\ln \tau_{1}\right)^{\sigma_{1}-1}\right|}{\Gamma\left(\sigma_{1}\right)}\right) \int_{1}^{e}\left(\ln \frac{e}{s}\right)^{\sigma_{1}-1} \frac{1}{s} d s \\
& \leq \frac{M_{h_{1}}}{\Gamma\left(\sigma_{1}\right)}\left|\int_{1}^{\tau_{1}}\left[\left(\ln \frac{\tau_{1}}{s}\right)^{\sigma_{1}-1}-\left(\ln \frac{\tau_{2}}{s}\right)^{\sigma_{1}-1}\right] \frac{1}{s} d s\right| \\
& +\frac{M_{h_{1}}}{\Gamma\left(\sigma_{1}\right)}\left|\int_{\tau_{1}}^{\tau_{2}}\left(\ln \frac{\tau_{2}}{s}\right)^{\sigma_{1}-1} \frac{1}{s} d s\right| \\
& +\frac{M_{h_{1}}}{\Gamma\left(\sigma_{1}\right)}\left|\left(\ln \tau_{2}\right)^{\sigma_{1}-1}-\left(\ln \tau_{1}\right)^{\sigma_{1}-1}\right| \int_{1}^{e}\left(\ln \frac{e}{s}\right)^{\sigma_{1}-1} \frac{1}{s} d s \\
& \rightarrow 0 \quad \text { as } \tau_{1} \rightarrow \tau_{2},
\end{aligned}
$$

uniformly for all $(\vartheta, v) \in \mathcal{E}$. Similarly we have

$$
\left|\mathcal{B}_{2}(\vartheta, v)\left(\tau_{1}\right)-\mathcal{B}_{2}(\vartheta, v)\left(\tau_{2}\right)\right| \rightarrow 0 \quad \text { as } \tau_{1} \rightarrow \tau_{2}
$$

uniformly for all $(\vartheta, v) \in S$. This shows that $\mathcal{B}(S) \subset \mathcal{E}$ is uniformly bounded, equicontinuous and compact, in view of Arzela-Ascoli theorem. Consequently, $\mathcal{B}$ is a compact and continuous operator on $\mathcal{E}$.

Step III. Now we prove that the third condition $\left(a_{3}\right)$ of Theorem 2.5 holds. Let $(\vartheta, v)$ and $(\bar{x}, \bar{y}) \in \mathcal{E}=\mathcal{X} \times \mathcal{X}$ be such that

$$
(\vartheta, v)=\left(\mathcal{A}_{1}(\vartheta, v) \mathcal{B}_{1}(\bar{x}, \bar{y}), \mathcal{A}_{2}(\vartheta, v) \mathcal{B}_{2}(\bar{x}, \bar{y})\right)
$$

Then, we have

$$
\begin{aligned}
|\vartheta(t)|= & \left|\mathcal{A}_{1}(\vartheta, v)(t) \mathcal{B}_{1}(\bar{x}, \bar{y})(t)\right| \\
\leq & \left|\mathcal{A}_{1}(\vartheta, v)(t) B_{1}(\bar{x}, \bar{y})(t)\right| \\
\leq & {\left[\left|f_{1}(t, \vartheta, v)-f_{1}(t, 0,0)\right|+\left|f_{1}(t, 0,0)\right|\right] } \\
& \times\left(\frac{1}{\Gamma\left(\sigma_{1}\right)} \int_{1}^{t}\left(\ln \frac{t}{s}\right)^{\sigma_{1}-1} \frac{h_{1}(s, \bar{x}(s), \bar{y}(s))}{s} d s\right. \\
& +(\ln t)^{\sigma_{1}-1}\left[\frac{m(\bar{x})}{f_{2}(e, m(\bar{x}), n(\bar{y}))}\right. \\
& \left.-\frac{1}{\Gamma\left(\sigma_{1}\right)} \int_{1}^{e}\left(\ln \frac{e}{s}\right)^{\sigma_{1}-1} \frac{h_{1}(s, \bar{x}(s), \bar{y}(s))}{s} d s\right] \\
\leq & {\left[L_{f_{1}}(\|\vartheta\|+\|v\|)+F_{0}\right] } \\
& \times\left(M_{1}+\frac{M_{h_{1}}}{\Gamma\left(\sigma_{1}\right)} \int_{1}^{t}\left(\ln \frac{t}{s}\right)^{\sigma_{1}-1} \frac{1}{s} d s\right. \\
& \left.+\frac{M_{h_{1}}}{\Gamma\left(\sigma_{1}\right)} \int_{1}^{e}\left(\ln \frac{e}{s}\right)^{\sigma_{1}-1} \frac{1}{s} d s\right) \\
\leq & {\left[L_{f_{1}}(\|\vartheta\|+\|v\|)+F_{0}\right]\left(\frac{2 M_{h_{1}}}{\Gamma\left(\sigma_{1}+1\right)}+M_{1}\right) . }
\end{aligned}
$$


Thus, by taking the supremum of the left-hand side, we get

$$
\|\vartheta\| \leq\left[L_{f_{1}}(\|\vartheta\|+\|v\|)+F_{0}\right]\left(\frac{2 M_{h_{1}}}{\Gamma\left(\sigma_{1}+1\right)}+M_{1}\right)
$$

Similarly, proceeding as before, we obtain

$$
\|v\| \leq\left[L_{f_{2}}(\|\vartheta\|+\|v\|)+G_{0}\right]\left(\frac{2 M_{h_{2}}}{\Gamma\left(\delta_{1}+1\right)}+M_{2}\right) .
$$

Adding inequalities (20) and (21), we obtain

$$
\begin{aligned}
\|\vartheta(t)\|+\|v(t)\| \leq & {\left[L_{f_{1}}(\|\vartheta\|+\|v\|)+F_{0}\right]\left(\frac{2 M_{h_{1}}}{\Gamma\left(\sigma_{1}+1\right)}+M_{1}\right) } \\
& +\left[L_{f_{2}}(\|\vartheta\|+\|v\|)+G_{0}\right]\left(\frac{2 M_{h_{2}}}{\Gamma\left(\delta_{1}+1\right)}+M_{2}\right) \\
\leq & \frac{F_{0}\left(\frac{2 M_{h_{1}}}{\Gamma\left(\sigma_{1}+1\right)}+M_{1}\right)+G_{0}\left(\frac{2 M_{h_{2}}}{\Gamma\left(\delta_{1}+1\right)}+M_{2}\right)}{1-\left(\left[L_{f_{1}}\left(\frac{2 M_{h_{1}}}{\Gamma\left(\sigma_{1}+1\right)}+M_{1}\right)\right]+\left[L_{f_{2}}\left(\frac{2 M_{h_{2}}}{\Gamma\left(\delta_{1}+1\right)}+M_{2}\right)\right]\right)} \\
\leq & \frac{F_{0}\left(\frac{2 M_{h_{1}}}{\Gamma\left(\sigma_{1}+1\right)}+M_{1}\right)+G_{0}\left(\frac{2 M_{h_{2}}}{\Gamma\left(\delta_{1}+1\right)}+M_{2}\right)}{1-\Omega} \\
\leq & \rho .
\end{aligned}
$$

As $\|(\vartheta, v)\|=\|\vartheta\|+\|v\|$, we have that $\|(\vartheta, v)\| \leq \rho$ and so condition $\left(a_{3}\right)$ of Theorem 2.5 holds.

Step IV. We now prove that $M_{\mathcal{B}}<1$. Consider

$$
\begin{aligned}
M_{B} & =\|\mathcal{B}(S)\| \\
& =\sup \{\|\mathcal{B}(\vartheta, v)\|:(\vartheta, v) \in S\} \\
& =\sup \left\{\left\|\mathcal{B}_{1}(\vartheta, v)\right\|+\left\|\mathcal{B}_{2}(\vartheta, v)\right\|:(\vartheta, v) \in S\right\} \\
& \leq \frac{2 M_{h_{1}}}{\Gamma\left(\sigma_{1}+1\right)}+M_{1}+\frac{2 M_{h_{2}}}{\Gamma\left(\delta_{1}+1\right)}+M_{2} \\
& <1 .
\end{aligned}
$$

From the above estimate, we conclude that condition $\left(a_{4}\right)$ of Theorem 2.5 also holds.

Thus, the operators $\mathcal{A}$ and $\mathcal{B}$ satisfy all the conditions of Theorem 2.5 and so, for the considered values of $h_{1}$ and $h_{2}$, the coupled system of equations (3) has at least one solution on $[1, e]$.

\section{An example}

Example 4.1 Consider the following coupled system of Hadamard fractional differential equations with boundary conditions:

$$
\begin{aligned}
& D^{3 / 2}\left(\frac{|\vartheta(t)|}{\frac{1}{2}(1+|\sin \vartheta(t)|)}\right)=\frac{|\vartheta(t)|}{(24+i+t)(1+|\vartheta(t)|+|v(t)|)}, \quad 1 \leq t \leq e, \\
& D^{3 / 2}\left(\frac{|v(t)|}{\frac{1}{2}(1+|\cos \vartheta(t)|)}\right)=\frac{|v(t)|}{(20+i+t)|\vartheta(t)|}, \quad 1 \leq t \leq e,
\end{aligned}
$$




$$
\begin{aligned}
& u(0)=0, \quad \vartheta(e)=\frac{1}{16} \sin \vartheta(\eta), \quad \eta \in(0,1) \\
& \nu(0)=0, \quad v(e)=\frac{1}{18} \cos v(\xi), \quad \xi \in(0,1) .
\end{aligned}
$$

Here

$$
\begin{aligned}
& L_{f_{1}}=L_{f_{2}}=\frac{1}{2}, \quad M_{1}=\frac{1}{16}, \\
& M_{2}=\frac{1}{18}, \quad M_{h_{1}}=\frac{1}{25}, \quad M_{h_{2}}=\frac{1}{21}, \\
& L_{f_{1}}\left(M_{1}+\frac{2 M_{h_{1}}}{\Gamma\left(\sigma_{1}+1\right)}\right)+L_{f_{2}}\left(M_{2}+\frac{2 M_{h_{2}}}{\Gamma\left(\delta_{1}+1\right)}\right) \simeq 0.124936655<1 .
\end{aligned}
$$

Hence, all the conditions of Theorem 3.3 are satisfied; consequently, the problem (22) and (23) has a solution on $[1, e]$.

\section{Conclusions}

We have presented the existence result for boundary value problems of coupled hybrid differential systems involving the Hadamard fractional derivative. The proof of the existence result is based on the fixed point theorem due to Dhage [10]. The present work can be extended to coupled systems of nonlinear fractional differential equations involving Caputo-Hadamard fractional derivative.

\section{Acknowledgements}

The authors express their gratitude to the referees for their helpful suggestions, which improved the final version of this paper.

\section{Funding}

Not applicable.

\section{Availability of data and materials}

Not applicable.

\section{Competing interests}

The authors declare that they have no competing interests.

\section{Authors' contributions}

All authors contributed equally to this manuscript. All authors read and approved the final manuscript.

\section{Author details}

'Department of Mathematics, Sona College of Technology, Salem, 636005 Tamil Nadu, India. ${ }^{2}$ Department of Mathematics, Sri Vasavi College, Erode, 638316 Tamil Nadu, India. ${ }^{3}$ Department of Mathematics, Cankaya University, Ankara, Turkey. ${ }^{4}$ Institute of Space Sciences, 06530 Magurele-Bucharest, Romania. ${ }^{5}$ Department of Medical Research, China Medical University Hospital, China Medical University, Taichung, Taiwan.

\section{Publisher's Note}

Springer Nature remains neutral with regard to jurisdictional claims in published maps and institutional affiliations.

Received: 1 December 2019 Accepted: 21 June 2020 Published online: 14 August 2020

\section{References}

1. Ahmad, B., Alghamdi, N., Alsaedi, A., Ntouyas, S.K.: A system of coupled multi-term fractional differential equations with three-point coupled boundary conditions. Fract. Calc. Appl. Anal. 22(3), 601-618 (2019)

2. Ahmad, B., Alsaedi, A., Ntouyas, S.K., Tariboon, J.: Hadamard-Type Fractional Differential Equations, Inclusions and Inequalities. Springer, Cham (2017)

3. Ahmad, B., Karthikeyan, P., Buvaneswari, K.: Fractional differential equations with coupled slit-strips type integral boundary conditions. AlMS Math. 4(6), 1596-1609 (2019)

4. Ahmad, B., Ntouyas, S.K.: Initial-value problems for hybrid Hadamard fractional differential equations. Electron. J. Differ. Equ. 2014, 161 (2014) 
5. Ahmad, B., Ntouyas, S.K.: Nonlocal boundary value problems for hybrid fractional differential equations and inclusions of Hadamard type. Fract. Differ. Calc. 5(2), 107-123 (2015)

6. Akorede, M.B., Arawomo, P.O.: Existence of positive solutions for a coupled system of nonlinear boundary value problems of fractional order with integral boundary conditions. Int. J. Appl. Math. 30(4), 273-288 (2017)

7. Alsaedi, A., Baleanu, D., Etemad, S., Rezapour, S.: On coupled systems of time-fractional differential problems by using a new fractional derivative. J. Funct. Spaces 2016, 4626940 (2016)

8. Alsulami, H.H., Ntouyas, S.K., Agarwal, R.P., Ahmad, B., Alsaedi, A.: A study of fractional-order coupled systems with a new concept of coupled non-separated boundary conditions. Bound. Value Probl. 2017, 68 (2017)

9. Baleanu, D., Jafari, H., Khan, H., Johnston, S.J.: Results for Mild solution of fractional coupled hybrid boundary value problems. Open Math. 13, 601-608 (2015)

10. Dhage, B.C.: On a fixed point theorem in Banach algebras with applications. Appl. Math. Lett. 18, 273-280 (2005)

11. Dhage, B.C.: Some variants of two basic hybrid fixed point theorems of Krasnoselskii and Dhage with applications. Nonlinear Stud. 25(3), 559-573 (2018)

12. Dhage, B.C., Dhage, S.B., Buvaneswari, K.: Existence of mild solutions of nonlinear boundary value problems of coupled hybrid fractional integro differential equations. J. Fract. Calc. Appl. 10(2), 191-206 (2019)

13. Hajipour, M., Jajarmi, A., Baleanu, D., Sun, H.G.: On an accurate discretization of a variable-order fractional reaction-diffusion equation. Commun. Nonlinear Sci. Numer. Simul. 69, 119-133 (2019)

14. Jena, R.M., Chakraverty, S., Baleanu, D.: On the solution of an imprecisely defined nonlinear time-fractional dynamical model of marriage. Mathematics 7(8), 689 (2019)

15. Karthikeyan, P., Buvaneswari, K.: A note on coupled fractional hybrid differential equations involving Banach algebra. Malaya J. Mat. 6(4), 843-849 (2018)

16. Khan, H., Li, Y., Chen, W., Baleanu, D., Khan, A.: Existence theorems and Hyers-Ulam stability for a coupled system of fractional differential equations with p-Laplacian operator. Bound. Value Probl. 2017, 157 (2017)

17. Kilbas, A.A.: Hadamard-type fractional calculus. J. Korean Math. Soc. 38, 1191-1204 (2001)

18. Kilbas, A.A., Srivastava, H.M., Trujillo, J.J.: Theory and Applications of Fractional Differential Equations. North-Holland Mathematical Studies., vol. 204. Elsevier, Amsterdam (2006)

19. Nageswara Rao, S., Alesemi, M.I.: On a coupled system of fractional differential equations with nonlocal non-separated boundary conditions. Adv. Differ. Equ. 2019, 97 (2019)

20. Samko, S.G., Kilbas, A.A., Marichev, O.I.: Fractional Integrals and Derivatives. Theory and Applications. Gordon \& Breach, Yverdon (1993)

21. Wang, J., Zhou, Y., Medved, M.: Existence and stability of fractional differential equations with Hadamard derivative. Topol. Methods Nonlinear Anal. 41, 113-133 (2013)

22. Wang, J.R., Zhang, Y.: On the concept and existence of solutions for fractional impulsive systems with Hadamard derivatives. Appl. Math. Lett. 39, 85-90 (2015)

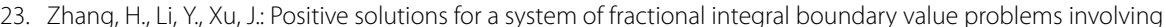
Hadamard type Fractional Derivatives. Hindawi Complex. 2019, Article ID 2671539 (2019)

24. Zhao, K., Suo, L., Liao, Y.: Boundary value problem for a class of fractional integro-differential coupled systems with Hadamard fractional calculus and impulses. Bound. Value Probl. 2019, 105 (2019)

\section{Submit your manuscript to a SpringerOpen ${ }^{\circ}$ journal and benefit from:}

- Convenient online submission

- Rigorous peer review

- Open access: articles freely available online

- High visibility within the field

- Retaining the copyright to your article

Submit your next manuscript at $\gg$ springeropen.com 